\title{
Identification of candidate substrates of ubiquitin-specific protease 13 using 2D-DIGE
}

\author{
JIANMIN WANG, YINGLI LIU, LIJUAN TANG, SUFEN QI, YINGJUN MI, DIANWU LIU and QINGBAO TIAN
}

\author{
Department of Epidemiology and Statistics, School of Public Health, \\ Hebei Medical University, Shijiazhuang, Hebei 050017, P.R. China
}

Received May 13, 2016; Accepted May 4, 2017

DOI: $10.3892 /$ ijmm.2017.2984

\begin{abstract}
The present study aimed to identify candidate substrates of ubiquitin-specific protease (USP)13 using two-dimensional fluorescence difference gel electrophoresis (2D-DIGE). USP13 is a well-characterized member of the USP family, which regulates diverse cellular functions by cleaving ubiquitin from ubiquitinated protein substrates. However, existing studies indicate that USP13 has no detectable hydrolytic activity in vitro. This finding implies that USP13 likely has different substrate specificity. In this study, a USP cleavage assay was performed using two different types of model substrates (glutathione S-transferase-Ub52 and ubiquitin- $\beta$-galactosidase) to detect the deubiquitinating enzyme (DUB) activity of USP13. In addition, a proteomic approach was taken by using 2D-DIGE to detect cellular proteins whose expressoin is significantly altered in 293T cell lines following the overexpression of USP13 or its C345S mutant (the catalytically inactive form). The data indicated that USP13 still has no detectable DUB activity in vitro nor does C345S. The results of 2D-DIGE demonstrated that the expression of several proteins increased or decreased significantly in $293 \mathrm{~T}$ cells following the overexpression of USP13. Mass spectroscopy analysis of gel spots identified 7 proteins, including 4 proteins with an increased expression, namely vinculin, thimet oligopeptidase, cleavage and polyadenylation specific factor 3 , and methylosome protein 50, and 3 proteins with a decreased expression, namely adenylosuccinate synthetase, annexin and phosphoglycerate mutase. In addition, in the samples of 293T cell lines after the overexpression of USP13 and USP13 C345S, vinculin exhibited an increased expression, suggesting that it may be a candidate
\end{abstract}

Correspondence to: Dr Qingbao Tian, Department of Epidemiology and Statistics, Hebei Medical University School of Public Health, 361 East Zhongshan Road, Shijiazhuang, Hebei 050017, P.R. China E-mail: tqb1980@hebmu.edu.cn

Key words: enzymatic activity, two-dimensional difference gel electrophoresis, liquid chromatography-mass spectroscopy/mass spectrometry, ubiquitin-specific protease 13 , vinculin substrate of USP13. However, sufficient follow-up validation studies are required in order to determine whether vinculin protein directly interacts with USP13.

\section{Introduction}

The ubiquitin-specific proteases (USPs) represent the most evolutionarily conserved and well represented deubiquitinating enzymes (DUBs) (1). They contain conserved Cys, His and Asp/Asn domains in their amino acid sequences (2), which include the residues critical for catalysis. Accordingly, USPs have the ability to remove ubiquitin from a ubiquitinated substrate, thereby regulating the localization and activity of this substrate (2-5). The ability of USPs to cleave model ubiquitin fusion protein substrates was previously investigated by co-expression in Escherichia coli (E.coli) (6). USP enzymes are characterized by the presence of two conserved active site domains, and several have been shown to cleave ubiquitin from model substrates, such as ubiquitin$\beta$-galactosidase (Ub-Met- $\beta$-gal) and pGEX-Ub52 [glutathione S-transferase (GST)-Ub52] (7), by hydrolyzing the bond between the C-terminal double glycine of ubiquitin and the linking methionine residue (6). However, ubiquitin C-terminal 7-amido-4-methylcoumarin (Ub-AMC) and its analogs, due to their high fluorescence sensitivity and hydrolysis efficiency, are often used to quantitatively determine the activities of DUBs without considering their substrate specificities $(8,9)$.

USP13, also named isopeptidase T-3, which was first identified by Timms et al (10), is a well-characterized member of USPs (11). USP13 shares a $54.8 \%$ identity with its isoform USP5 $(12,13)$. Both proteins contain 4 ubiquitin-associated (UBA) domains and an ubiquitin-specific processing protease (UBP) domain. The UBP domain of USP13 harbors a catalytic site, a zinc finger (ZnF) domain, and 2 UBA domains. Domains such as UBA and $\mathrm{ZnF}$ play very important roles in USP13 (14). The UBA domain usually facilitates substrate binding onto the enzymes, while the $\mathrm{ZnF}$ domain may be an activator to stimulate the hydrolysis reaction or a regulator to orchestrate other biological functions (15). Although USP13 contains all 4 putative ubiquitin-binding domains, it has not been shown to hydrolyze polyubiquitin (16), but rather serves as a protease for interferon-stimulated gene 15 (ISG15), whereby it reduces ISGylation (16). A previous study indicated that a few residues in USP13-ZnF vary, particularly in the L2-loop region, 
in which R221 and Y261 in USP5-ZnF are the key residues for ubiquitin binding and catalytic activation (13). The $\mathrm{ZnF}$ domain of USP13 cannot bind with ubiquitin, so that USP13 loses its ability to be activated by free ubiquitin (14). However, USP13 exhibits a weak deubiquitinating activity for Lys63-linked polyubiquitin (K63-polyUb) in a non-activating manner, and it cannot be activated by the reaction product (14). Nevertheless, in a recent study, the ability of USP13 to bind free ubiquitin and cleave ubiquitin-AFC (Ub-AFC) was tested in vitro, and USP13 was almost completely inactive and could not efficiently cleave K48- or K63-linked di-ubiquitin (17). Thus, the catalytic function and the regulatory mechanism of USP13 remain to be defined.

In addition to studies on the enzymatic activity of USP13, elucidating the substrate specificities of USP13 is vital to understanding its biological function. It has recently been demonstrated that USP13 can regulate Siah2 ligase stability and activity via non-catalytic ubiquitin-binding domains (18). It can also deubiquitinate signal transducer and activator of transcription 1 (STAT1) and increase STAT1 protein stability (19), reduce ubiquitination and degradation of Sky2 (20), and also appears to be responsible for the protein stability of microphthalmia-associated transcription factor (MITF), which is essential for the development of melanocytes (21). Liu et al also supported the existence of an interactive regulatory association between USP10 and 13 with Vps34 complexes (22). Furthermore, USP13 was identified as the first deubiquitylase that reverses phosphatase and tensin homolog (PTEN) polyubiquitylation and stabilizes PTEN protein in human breast cancer cells (23). Recently, a new study found that USP13 interacted with PTEN in fibroblasts and regulated PTEN ubiquitination and degradation (24). Another study revealed an unexpected interaction between USP13 and ERAD-specific ubiquitin ligase gp78, thereby promoting ERAD (17). In addition to these findings, further research is required to identify additional substrates of USP13.

This study confirmed that USP13 proteins have no detectable DUB activity detected by USP cleavage assay using two different types of model substrates (GST-Ub52 and Ub-Met- $\beta$-gal). In addition, a proteomics approach was taken by using two-dimensional fluorescence difference gel electrophoresis (2D-DIGE) to detect cellular proteins with significantly altered expression levels in $293 \mathrm{~T}$ cells following the overexpression of USP13. As a specificity control, this study also aimed to examine the effect of a catalytically inactive mutant of USP13. To establish such a mutant, the amino acid sequence of USP13 was aligned to USP5, a DUB with a well-defined cysteine catalytic motif, and thereby cysteine 345 was identified as the corresponding catalytically critical residue, mutating it to serine (2). Several proteins were detected and identified using liquid chromatography-mass spectroscopy (LC-MS)/MS. Among the identified proteins, the altered expression level of vinculin was associated with the DUB activity of USP13, which was confirmed by western blot analysis and reverse transcription-quantitative polymerase chain reaction (RT-qPCR).

\section{Materials and methods}

Plasmid construction and site-directed mutagenesis. The plasmid pGEX-USP13 (wild-type), which expresses the GST-
USP13 fusion protein, was constructed by inserting the DNA fragment encoding the corresponding open reading frames between the SalI and NotI (both from New England Biolabs, Hitchin, UK) sites of plasmid pGEX-6p-1. A catalytically inactive version of USP13, pGEX-USP13 C345S (cysteine 345 mutated to serine), was then constructed by site-directed mutagenesis with the following primer sequences: 5'-AGCTATCTCAGCTCTGTCATG-3' and 5'-GCTG TTGCCCAGGTTCTTCAG-3'. A pAC-T7-USP13 plasmid was produced by inserting the complete coding sequence of USP13 into the pAC-T7 plasmid at the BamHI site. The pAC-T7 plasmid was produced according to the method described in the study by Zhang et al (7). To generate the plasmids pEGFP-USP13 and pEGFP-USP13 C345S, the pGEX-USP13 and pGEX-USP13 C345S vectors were digested with SalI and NotI (both from New England Biolabs), respectively, and then inserted into the pEGFP-C1 vector, which was linearized by EcoRI (New England Biolabs).

Expression and USP cleavage assay. E. coli $\mathrm{DH} 5 \alpha$ harboring pGEX-USP13 was incubated in Luria-Bertani broth medium at 37 or $15^{\circ} \mathrm{C}$ overnight to express GST-USP13 fusion proteins. GST fusion proteins were purified using GSH-Sepharose resin (Sangon Biotech Co., Ltd., Shanghai, China) and detected using $10 \%$ sodium dodecyl sulfate-polyacrylamide gel electrophoresis (SDS-PAGE).

The USP cleavage assays used Ub-Met- $\beta$-gal and GST-Ub52 as model substrates, as previously described (7). For cleavage of the GST-Ub52 substrate, E. coli strain BL21 (DE3) cells harboring pGEX-Ub52 were further transformed with pACT7-USP13 or pAC-T7-USP46 [plasmids expressing USP46 were used as positive controls (25)]. The protein expression was induced by isopropyl $\beta$-D-1-thiogalactopyranoside (IPTG). Soluble protein extracts were prepared after sonicating the cell lysate. GST fusion proteins and their cleavage products were purified using GSH-Sepharose resin (Sangon Biotech Co., Ltd.) and detected by $10 \%$ SDS-PAGE. The USP cleavage assay using Ub-Met- $\beta$-gal as a model substrate has been previously described (26). E. coli BL21 (DE3) bacteria harboring pGEX-USP13 or pGEX-USP46 [plasmids expressing USP46 were used as positive controls (27)] were transformed with pAC-M- $\beta$-gal, AmpR CmR colonies were grown and induced with IPTG, and total protein extracts were analyzed by western blotting with anti- $\beta$-gal antibody (Z3781; Promega, Madison, WI, USA). Total protein extracts were examined by western blot analysis with anti-T7 purified polyclonal antibody (AB3790; Millipore, Billerica, MA, USA) and anti-GST antibody (ab111947; Abcam, Cambridge, UK) to detect the expression of USPs.

Cell culture and transient transfection. The 293T cell line was purchased from the American Type Culture Collection (ATCC, Manassas, VA, USA). The cell line was seeded in a 6-well plate at a concentration of $4 \times 10^{5}$ cells $/ 2 \mathrm{ml}$ and cultured in Dulbecco's modified Eagle's medium (DMEM) supplemented with $10 \%$ fetal calf serum (FCS) (both from Gibco Life Technologies, Grand Island, NY, USA) and $1 \%$ penicillin-streptomycin at $37^{\circ} \mathrm{C}$ in a $5 \% \mathrm{CO}_{2}$ atmosphere. After $24 \mathrm{~h}$, the medium was replaced with fresh medium without antibiotics. The purified recombinant plasmids, pEGFP-C1, pEGFP-USP13 and pEGFP-USP13 
C345S, were transfected into the cells using Lipofectamine 2000 transfection reagent (Invitrogen Life Technologies, Carlsbad, CA, USA) according to the manufacturer's instructions. Following incubating for $24 \mathrm{~h}$, the cells were collected by centrifugation $\left(1,000 \mathrm{x} \mathrm{g}\right.$ for $5 \mathrm{~min}$ at $\left.4^{\circ} \mathrm{C}\right)$.

\section{D-DIGE}

Protein isolation and sample preparation. The proteins were extracted from the cells transfected with the pEGFP-USP13, pEGFP-USP13 C345S or pEGFP-C1 plasmids using SDS extraction buffer (100 mM Tris- $\mathrm{HCl}, \mathrm{pH}$ 8.0, 2\% SDS, $1 \% \beta$-mercaptoethanol, $5 \mathrm{mM}$ ethylene glycol tetraacetic acid, $10 \mathrm{mM}$ ethylenediaminetetraacetic acid, $1 \mu \mathrm{M}$ E-64, $1 \mu \mathrm{M}$ bestatin, $1 \mu \mathrm{M}$ pepstatin, $2 \mu \mathrm{M}$ leupeptin, $1 \mathrm{mM}$ phenylmethylsulfonyl fluoride and protease inhibitor cocktail and phosphatase inhibitor). The supernatants of the cell lysates were extracted using an equivalent volume of ice-cold Tris-saturated phenol ( $\mathrm{pH} 8.0)$, and then precipitated overnight with 5 volumes of chilled $0.1 \mathrm{M}$ ammonium acetate in methanol at $-20^{\circ} \mathrm{C}$. After rinsing twice with ice-cold ammonium acetate in methanol, followed by 2 rinses with chilled methanol, the pellets were solubilized in DIGE buffer [7M urea, 2M thiourea, 4\% 3-[(3-(cholamidopropyl) dimethylammonio]-1-propanesulfonate (CHAPS), $\mathrm{pH}$ 8.8]. The total protein concentrations were measured using the Bradford method (28) and adjusted to $5 \mu \mathrm{g} / \mu \mathrm{l}$.

Protein labeling. 2D-DIGE was performed following the method described in the study by Tang (29). For protein labeling, $50 \mathrm{pmol}$ of CyDye was mixed with $10 \mu \mathrm{l}$ of protein sample and combined with Cy3- and Cy5-labeled control and treated samples. Total protein extracts of USP13 were labeled with $\mathrm{Cy} 5$ in the treated samples, whereas the EGFP proteins were labeled with $\mathrm{Cy} 3$ in the control samples. The proteins were then pooled together with the treated samples prior to 2D-DIGE. The samples were incubated on ice for at least $2 \mathrm{~h}$ in the dark. The labeling reaction was terminated by the addition $1 \mu \mathrm{l}$ of $10 \mathrm{mM}$ lysine. For analytical 2D-DIGE analysis, $20 \mu \mathrm{l}$ of combined Cy3-or Cy5-labeled protein was mixed with $2 \mathrm{D}$-DIGE buffer $(7 \mathrm{M}$ urea, $2 \mathrm{M}$ thiourea, 4\% CHAPS, $20 \mathrm{mM}$ dithiothreitol, $0.5 \%$ IPG buffer) and used for isoelectric focusing (IEF). IEF was performed on 24-cm IPG strips, pH 4.0-7.0. The second-dimension electrophoresis was performed using 10\% SDS-polyacrylamide gels. The 2D gels were then scanned on a Typhoon 9410 imager and DIGE images were analyzed using DeCyder 6.5 software (both from GE Healthcare Life Sciences, Logan, UT, USA), and spot detection was performed using the differential in-gel analysis module and the DeCyder biological variation analysis module. Protein spots displaying an average $\geq 1$.1-fold change in abundance between the control and treated samples with a value of $\mathrm{P}<0.05$ were selected for picking on an Ettan Spot Picker (GE Healthcare Life Sciences). For each treatment, images from at least 3 biological repeats were used for the statistical analysis of protein abundance.

$L C-M S / M S$. Protein spots were excised from the gels and sent to the Department of Neuroplasticity of Shinshu University Graduate School of Medicine in Japan for LC-MS/MS analysis.

Mass spectra were subjected to Mascot search engine for protein identification. Database searching was performed using the Mascot (http://www.matrixscience.com) program.
$R T-q P C R$. Total RNA from the cells transfected with pEGFPUSP13, pEGFP-USP13 C345S, or pEGFP-C1 was extracted using TRIzol reagent (Invitrogen Life Technologies) and reverse-transcribed into cDNA using a RevertAid First Strand cDNA Synthesis kit (Thermo Fisher Scientific, Inc., Waltham, MA, USA). PCR quantification involved the use of TransStart Top Green qPCR SuperMix (TransGen Biotech Co., Ltd., Beijing, China) with primer sequences for vinculin (5'-AATG AGAGGGCAGTGTTTCC-3' and 5'-GGATGTTCAGGCAA GGTTCT-3'). The mRNA levels of vinculin, as well as those of the internal standard $\beta$-actin (Sangon Biotech Co., Ltd.), were measured by qPCR in triplicate using a Rotor Gene 6000 real-time detection system (Bio-Rad Laboratories, Inc., Hercules, CA, USA).

Western blot analysis. Total protein extracts of the cells transfected with pEGFP-USP13, pEGFP-USP13 C345S or pEGFP-C1 were examined by western blot analysis with vinculin antibody (OAAN00512; AVIVA Systems Biology, San Diego, CA, USA) to detect the expression of vinculin. With $\beta$-actin being an internal reference gene with anti$\beta$-actin (Proteintech Group, Inc., Chicago, IL, USA), the relative intensity of the resulting bands was analyzed using Odyssey V3.0 software (Odyssey, Lincoln, NE, USA).

Statistical analysis. All statistical analyses were performed using SPSS software version 17.0 (SPSS, Inc., Chicago, IL, USA). Statistical analysis was performed using the Student's t-test and one-way analysis of variance (ANOVA). A P-value $<0.05$ was considered to indicate a statistically significant difference.

\section{Results}

Low temperature improves the solubility of GST-USP13 recombinant proteins. The nucleotide sequence of homo USP13 has been submitted to GenBank under the accession no. HM138083. GST-USP13 fusion proteins of approximately $120 \mathrm{kDa}$ were expressed in E. coli BL21 and detected by $10 \%$ SDS-PAGE. Homo USP13 protein fused with GST was expressed in E. coli strain BL21 and induced by IPTG at 37 and $15^{\circ} \mathrm{C}$. When induced at $37^{\circ} \mathrm{C}$ for $4 \mathrm{~h}$, a major part of GST-USP13 precipitated; on the contrary, GST-USP13 fusion protein induced with IPTG at $15^{\circ} \mathrm{C}$ mostly existed in the supernatant (Fig. 1), thereby facilitating the purification of the fusion proteins for further analyses. GST fusion proteins were purified by GSH-Sepharose resin (Sangon Biotech Co., Ltd.) (Fig. 1B) and used to measure the hydrolytic activities of recombinant GST-fused USP13 (100 nM) using Ub-AMC as a substrate (data not shown).

USP13 exhibits no detectable hydrolytic activity for model substrates. To determine whether USP13 has DUB activity, a USP cleavage assay was performed using GST-Ub52 as a model substrate. USP46 cleaved GST-Ub52 efficiently to yield a product of the expected size of $36 \mathrm{kDa}$ as a positive control (Fig. 2C). Although western blot analysis with anti-T7 antibody can detect the expression of T7-USP13 (Fig. 2B), the hydrolytic activity of USP13 for GST-Ub52, as well as the empty vector alone and the C345S mutant, was not detected (Fig. 2C). 
A

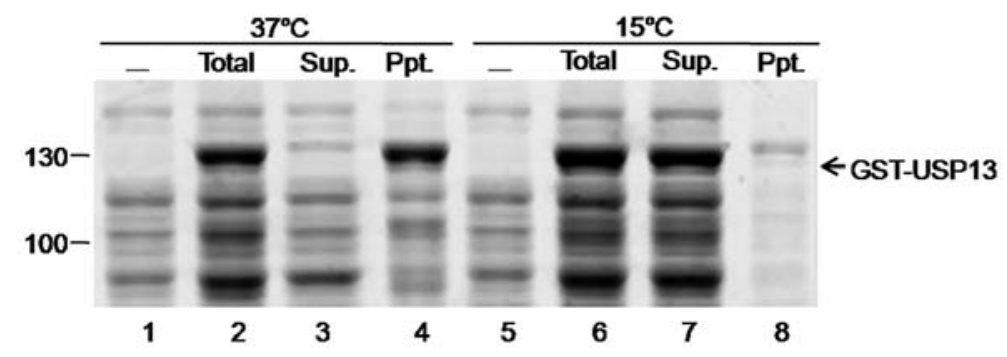

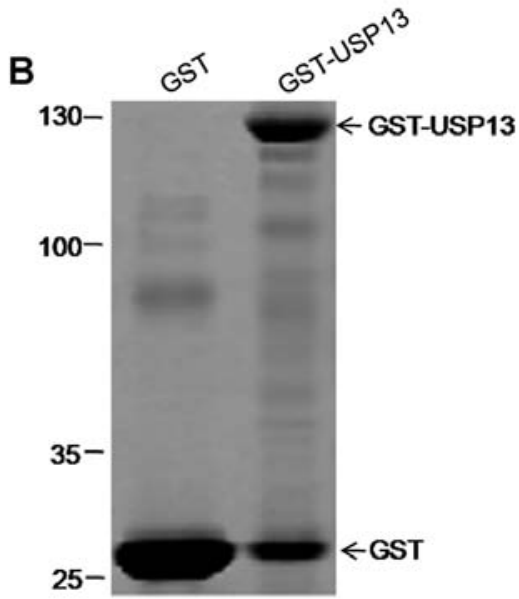

Figure 1. The expression and solubility of glutathione S-transferase (GST)-ubiquitin-specific protease 13 (USP13) fusion protein at different temperatures. (A) Homo USP13 protein fused with GST was expressed in Escherichia coli (E. coli) strain BL21 and induced by isopropyl $\beta$-D-1-thiogalactopyranoside (IPTG) at 37 and $15^{\circ} \mathrm{C}$. Bacterial lysate after ultrasonic treatment and centrifugation into different components: lanes 4 and 8, precipitate (Ppt.); lanes 3 and 7, supernatant (Sup.). Different components were detected by 10\% SDS-PAGE. (B) GST and GST-USP13 fusion protein were purified by glutathione-agarose beads and detected by $10 \%$ SDS-PAGE.

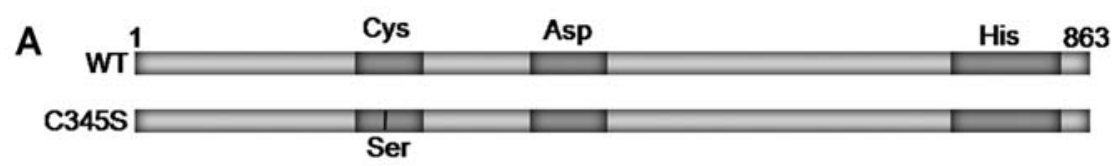
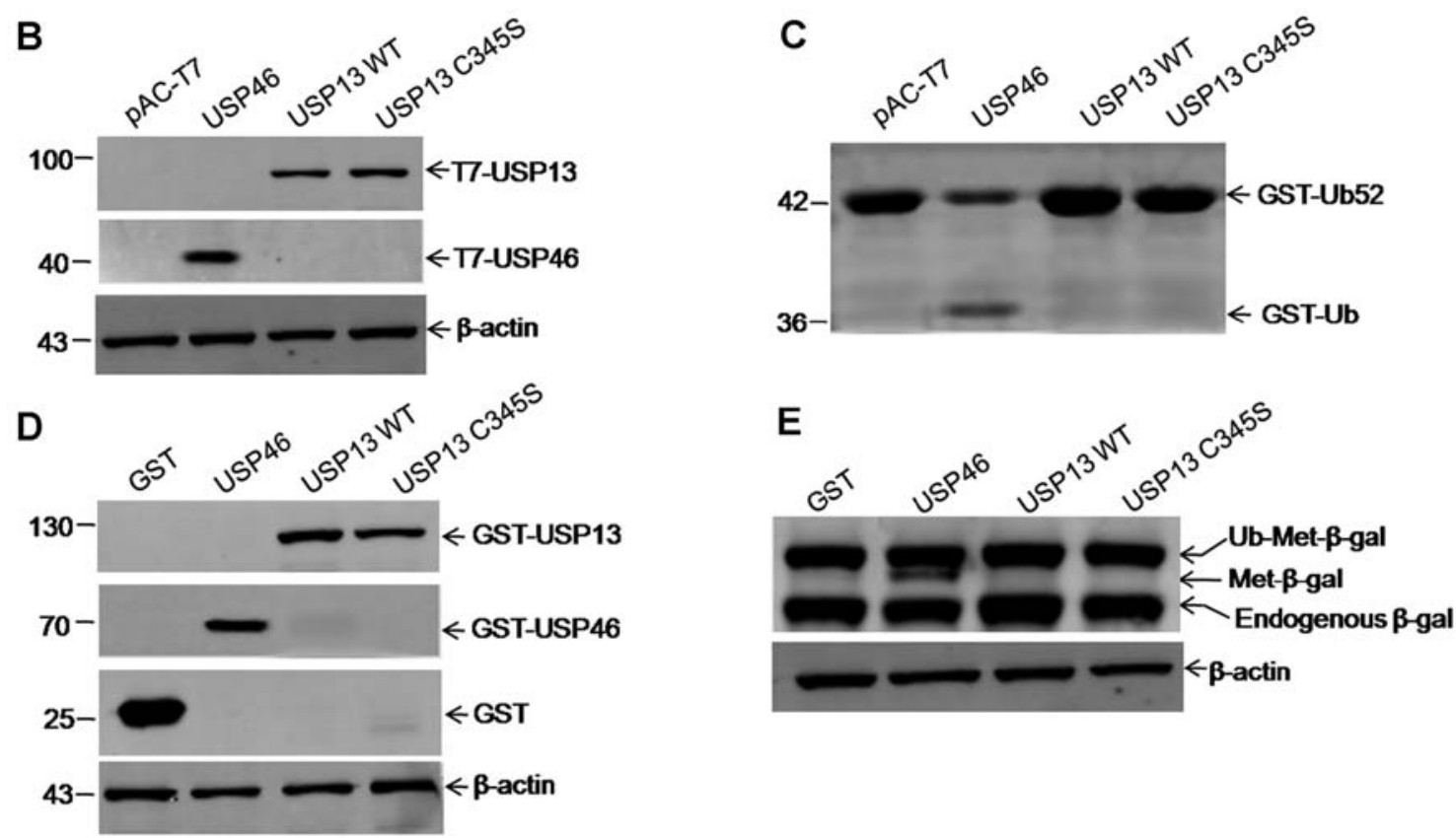

Figure 2. Ubiquitin-specific protease 13 (USP13) exhibited no detectable activity for the hydrolysis of model substrates. (A) Constructs of wild-type (WT) and mutant USP13 C345S for the USP cleavage assay. (B) Expression of T7-USP13 and T7-USP46 can be detected by western blotting with anti-T7 antibody. (C) Cleavage of model substrate glutathione S-transferase (GST)-Ub52. The arrows indicate the GST-Ub52 (about $42 \mathrm{kDa}$ ) and its cleavage products GST-Ub (about $36 \mathrm{kDa}$ ), which were detected by 10\% SDS-PAGE. USP46 and pAC-T7 (an empty plasmid vector) are used as positive and negative controls, respectively. (D) Total protein extracts were examined by western blot analysis with anti-GST to detect the expression of USPs. (E) Deubiquitination of Ub-Met- $\beta$-gal expressed in bacteria. Western blot analysis with anti- $\beta$-gal antiserum is shown. The arrows indicate the ubiquitin- $\beta$-galactosidase (Ub-Met$\beta$-gal) fusion protein, the cleaved $\beta$-gal moiety (Met- $\beta$-gal), and endogenous $\beta$-gal fragments. Co-expressed plasmids were pGEX-USP13, the positive control pGEX-USP46, and the negative control pGEX-6P-1 (GST).

The hydrolytic activities of recombinant GST-fused USP13 were further examined using Ub-Met- $\beta$-gal as a substrate, and
USP13 exhibited no detectable activity either (Fig. 2D and E). The hydrolytic activities of recombinant GST-fused 
Table I. Summary of the proteins identified by LC-MS/MS.

\begin{tabular}{|c|c|c|c|c|c|c|c|c|c|}
\hline \multirow{2}{*}{$\begin{array}{l}\text { Master } \\
\text { no. }{ }^{\text {a }}\end{array}$} & \multirow{2}{*}{$\begin{array}{l}\text { Accession } \\
\text { no. }\end{array}$} & \multirow{2}{*}{$\begin{array}{l}\text { Protein } \\
\text { name }\end{array}$} & \multicolumn{2}{|c|}{ Theoretical } & \multirow{2}{*}{$\begin{array}{l}\text { Protein } \\
\text { score }\end{array}$} & \multirow{2}{*}{$\begin{array}{l}\text { Protein } \\
\text { matched } \\
\text { peptides }\end{array}$} & \multirow{2}{*}{$\begin{array}{c}\text { Protein } \\
\text { sqeCover }(\%)\end{array}$} & \multirow{2}{*}{$\begin{array}{l}\text { Avg } \\
\text { ratio }^{\mathrm{a}}\end{array}$} & \multirow[b]{2}{*}{$\mathrm{P}$-value ${ }^{\mathrm{b}}$} \\
\hline & & & Size (Da) & $\mathrm{pI}$ & & & & & \\
\hline $1 / \mathrm{I}$ & P18206 & Vinculin & $116,722.62$ & 5.90 & $5,615.65$ & 81 & 84.02 & $1.23 / 1.21$ & $0.008 / 0.010$ \\
\hline 2 & P52888 & $\begin{array}{l}\text { Thimet } \\
\text { Oligopeptidase (THOP1) }\end{array}$ & $78,840.00$ & 5.87 & $1,693.12$ & 13 & 37.48 & 1.21 & 0.008 \\
\hline 3 & G5E9W3 & $\begin{array}{l}\text { Cleavage and } \\
\text { polyadenylation specific } \\
\text { factor } 3(\mathrm{CPSF} 3)\end{array}$ & $77,486.24$ & 5.42 & $1,053.86$ & 19 & 38.31 & 1.16 & 0.008 \\
\hline 4 & Q5T6W5 & $\begin{array}{l}\text { SH3 domain } \\
\text { containing RING } \\
\text { finger protein } 3\end{array}$ & $93,973.91$ & 9.99 & $1,580.02$ & 11 & 25.81 & 1.15 & 0.019 \\
\hline $5 / \mathrm{II}^{\mathrm{c}}$ & B3GQS7 & & & & & & & $1.32 / 1.31$ & $0.017 / 0.011$ \\
\hline $6 / \mathrm{III}^{\mathrm{c}}$ & F5GWR2 & & & & & & & $1.16 / 1.15$ & $0.02 / 0.024$ \\
\hline 7 & Q9BQA1 & $\begin{array}{l}\text { Methylosome } \\
\text { protein } 50 \text { (WDR77) }\end{array}$ & $36,724.50$ & 4.95 & $2,015.87$ & 20 & 61.25 & 1.17 & 0.013 \\
\hline 8 & F8W9D6 & $\begin{array}{l}\text { Adenylosuccinate } \\
\text { synthetase (ADSS) }\end{array}$ & $48,338.13$ & 6.37 & $12,416.72$ & 39 & 89.20 & -1.16 & 0.016 \\
\hline 9 & E7EW96 & Annexin & $38,714.27$ & 6.86 & $5,310.28$ & 16 & 79.14 & -1.2 & 0.014 \\
\hline 10 & P17987 & $\begin{array}{l}\text { T complex protein } 1 \\
\text { subunit } \alpha\end{array}$ & $43,885.80$ & 7.67 & 751.85 & 17 & 44.11 & -1.15 & 0.004 \\
\hline 11 & Q6P6D7 & $\begin{array}{l}\text { Phosphoglycerate } \\
\text { mutase (PGAM) }\end{array}$ & $28,776.89$ & 6.46 & $39,293.07$ & 13 & 55.12 & -1.23 & 0.010 \\
\hline
\end{tabular}

${ }^{\mathrm{a}} 1,5,6$ indicate the spots following USP13 overexpression compared with EGFP, and I, II, III indicate the spots following USP13 overexpression compared with USP13 C345S; ${ }^{b}$ The average ratio of gel spots in 2D-DIGE, P-value was determined by the Student's t-test; 'failure to identify. LC-MS, liquid chromatography-mass spectroscopy; 2D-DIGE, two-dimensional difference gel electrophoresis; USP13, ubiquitin-specific protease 13.

USP13 (100 $\mathrm{nM})$ were also measured using Ub-AMC as a substrate, and similar results were obtained (data not shown).

Screened proteins associated with the overexpression of USP13 by 2D-DIGE and LC-MS/MS. To determine whether USP13 alters cellular protein expression, a proteomic approach by 2D-DIGE was taken to detect cellular proteins with significantly altered expression levels in 293T cell lines following the overexpression of USP13.293T cells were transfected with an expression vector for USP13, USP13 C345S or an empty vector. Total protein extracts were labeled with $\mathrm{Cy} 5$ or $\mathrm{Cy} 3$ and then pooled together with the treated samples prior to 2D-DIGE. DeCyder analysis of these replicates detected approximately 2,500 spots in at least 2 of the 3 gels. For each treatment, images from at least 3 biological repeats were used for the statistical analysis of protein abundance.

A total of 11 spots displayed an average $\geq 1$.1-fold change in abundance with a value of $\mathrm{P}<0.05$ following the overexpression of USP13 compared with that of EGFP (Fig. 3A-C). The arrowheads and numbers indicate the spots with an increased and decreased expression. A total of 7 of the 11 species were identified using LC-MS/MS. These proteins included 4 proteins with an increased expression, namely vinculin, thimet oligopeptidase 1 (THOP1), cleavage and polyadenylation specific factor 3 (CPSF3) and methylosome protein 50 (WDR77), and 3 proteins with a decreased expression, namely adenylosuc- cinate synthetase (ADSS), annexin and phosphoglycerate mutase (PGAM), as shown in Fig. 3D and Table I. With regard to the remaining four spots, spots 4 and 10 were identified as SH3 domain containing RING finger protein 3 and T complex protein 1 subunit $\alpha$ (TCP1), respectively, with isoelectric points of 9.99 and 7.67 (Table I). These isoelectric points were beyond the range of the IPG strip used. Spots 5 and 6 could not be identified (Table I). The reasons for the failure to identify the other species may include problems in peptide elution, low amounts of protein, or scoring below the threshold in the Mascot search engine.

To investigate whether USP13 DUB activity affects the expression of these proteins, the USP13 C345S proteins were labeled with $\mathrm{Cy} 3$ in the control samples and pooled together with the treated samples prior to 2D-DIGE. USP13 C345S completely abolished its DUB activity (14). Three of the 11 spots still displayed an average $\geq 1$.1-fold increase in abundance with $\mathrm{P}<0.05$ (Fig. 4A-C). Following LC-MS/MS analysis, only vinculin exhibited an increased expression as a result of the expression of USP13 (Fig. 4D). Fig. 5 shows the threedimensional plots of spot density that increased in amount in 293T cell lines expressing USP13 and USP13 C345S, where the $\mathrm{z}$-axis (vertical dimension) shows the fluorescence signal intensity in that channel. These results suggest that the regulation of vinculin protein levels by USP13 may depend on the enzymatic activity of USP13. 

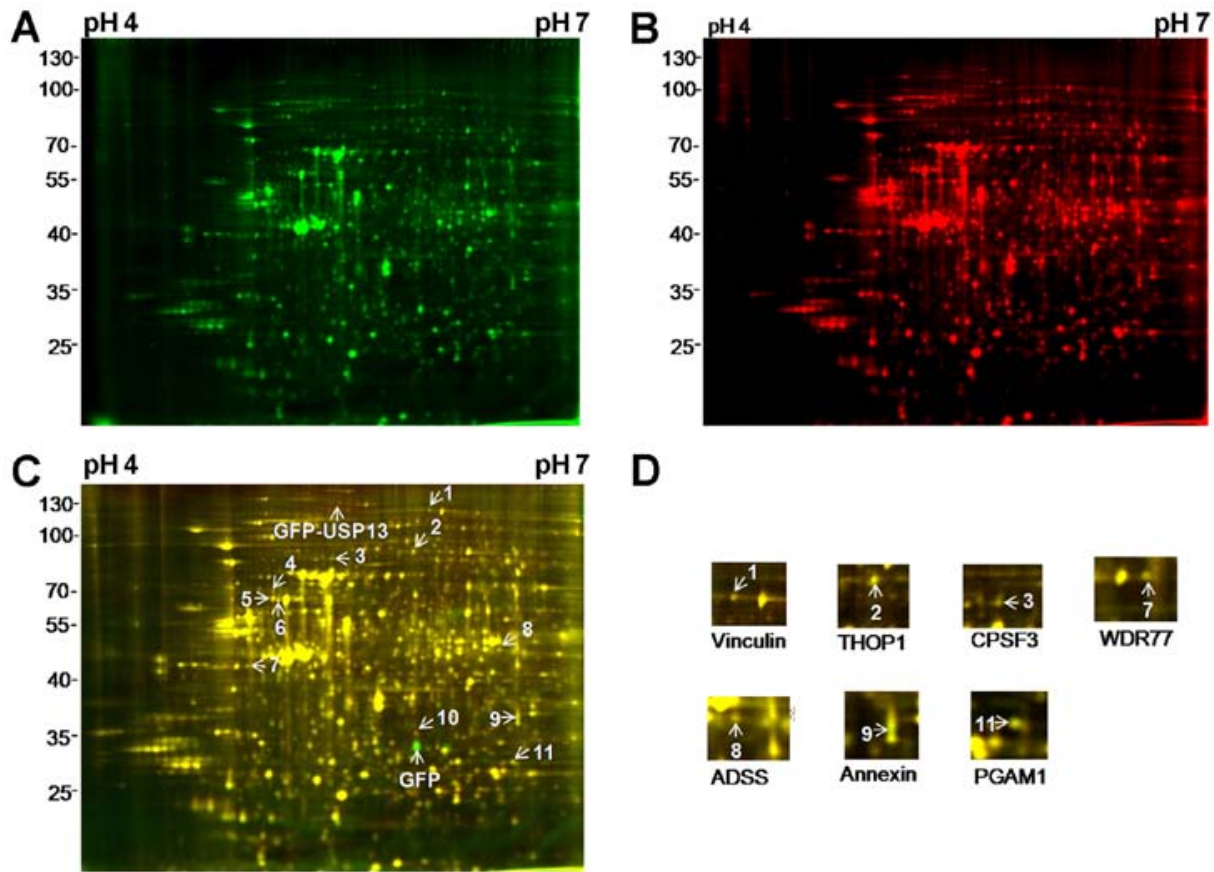

D 7

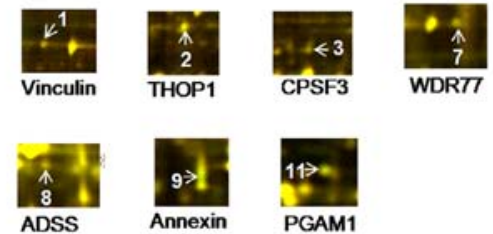

Figure 3. Screened proteins associated with the overexpression of ubiquitin-specific protease 13 (USP13) by two-dimensional fluorescence difference gel electrophoresis (2D-DIGE). (A) Proteins from 293T cell lines transfected with plasmid pEGFP-C1 labeled with Cy3 (green) as the control group. (B) Proteins from 293 T cell lines transfected with purified recombinant plasmid pEGFP-USP13 labeled with Cy5 (red) as the treated group. (C) The merged image showing Cy3- and Cy5-labeled proteins. The arrowheads and numbers indicate the spots with increased and decreased expression. (D) Seven proteins exhibited altered expression levels identified by liquid chromatography-mass spectroscopy (LC-MS)/MS.

A

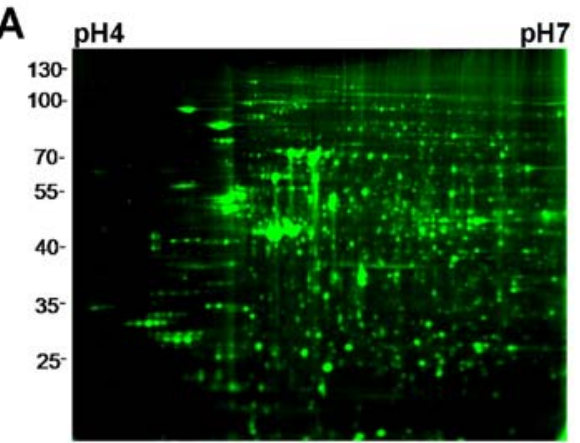

C

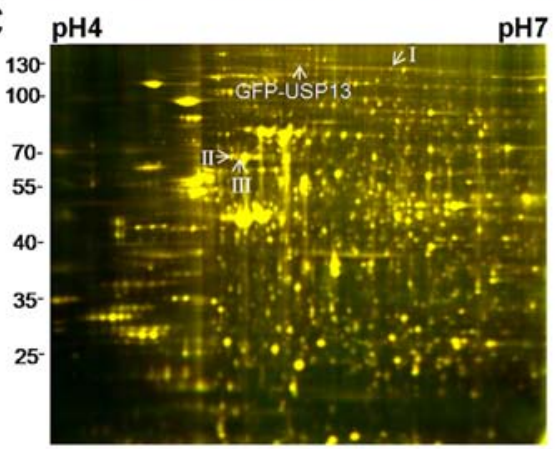

B
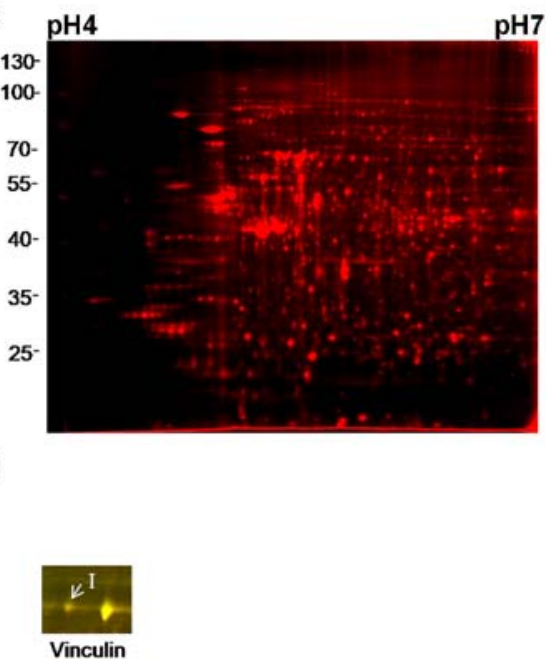

Figure 4. Screened proteins associated with the overexpression of ubiquitin-specific protease 13 (USP13) and USP13 C345S by two-dimensional fluorescence difference gel electrophoresis (2D-DIGE). (A) Proteins from 293 T cell lines transfected with plasmid pEGFP-USP13 C345S labeled with Cy3 (green) as the control group. (B) Proteins from $293 \mathrm{~T}$ cell lines transfected with purified recombinant plasmid pEGFP-USP13 labeled with Cy5 (red) as the treated group. (C) The merged image showing Cy3- and Cy5-labeled proteins. The arrowheads and numbers indicate the spots with increased and decreased expression. (D) Only one protein exhibited altered expression levels identified by liquid chromatography-mass spectroscopy (LC-MS)/MS.

USP13 upregulates vinculin protein levels, but not $m R N A$ levels. To further validate the upregulation of vinculin protein levels by USP13, western blot analysis and RT-qPCR analysis were performed. the 293T cells were transfected with an expression vector for USP13, USP13 C345S, or an empty vector (pEGFP-C1). At $24 \mathrm{~h}$ following transfection, total 
Spot \#
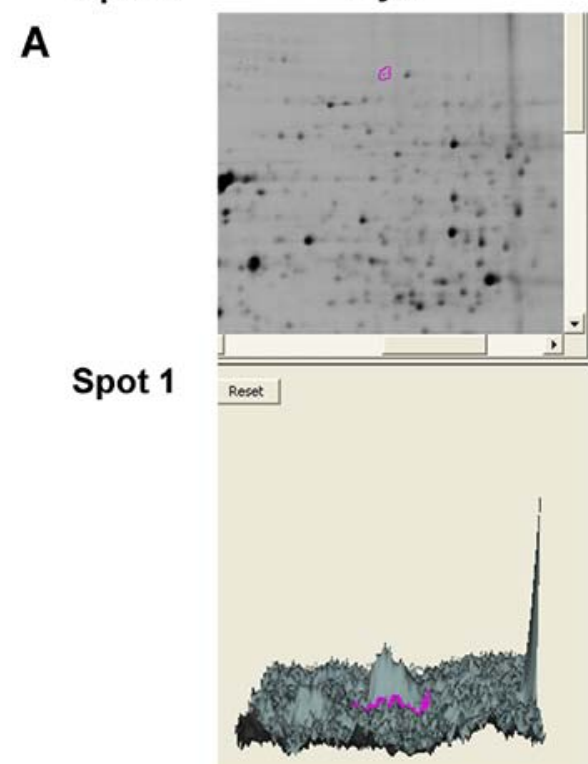

B
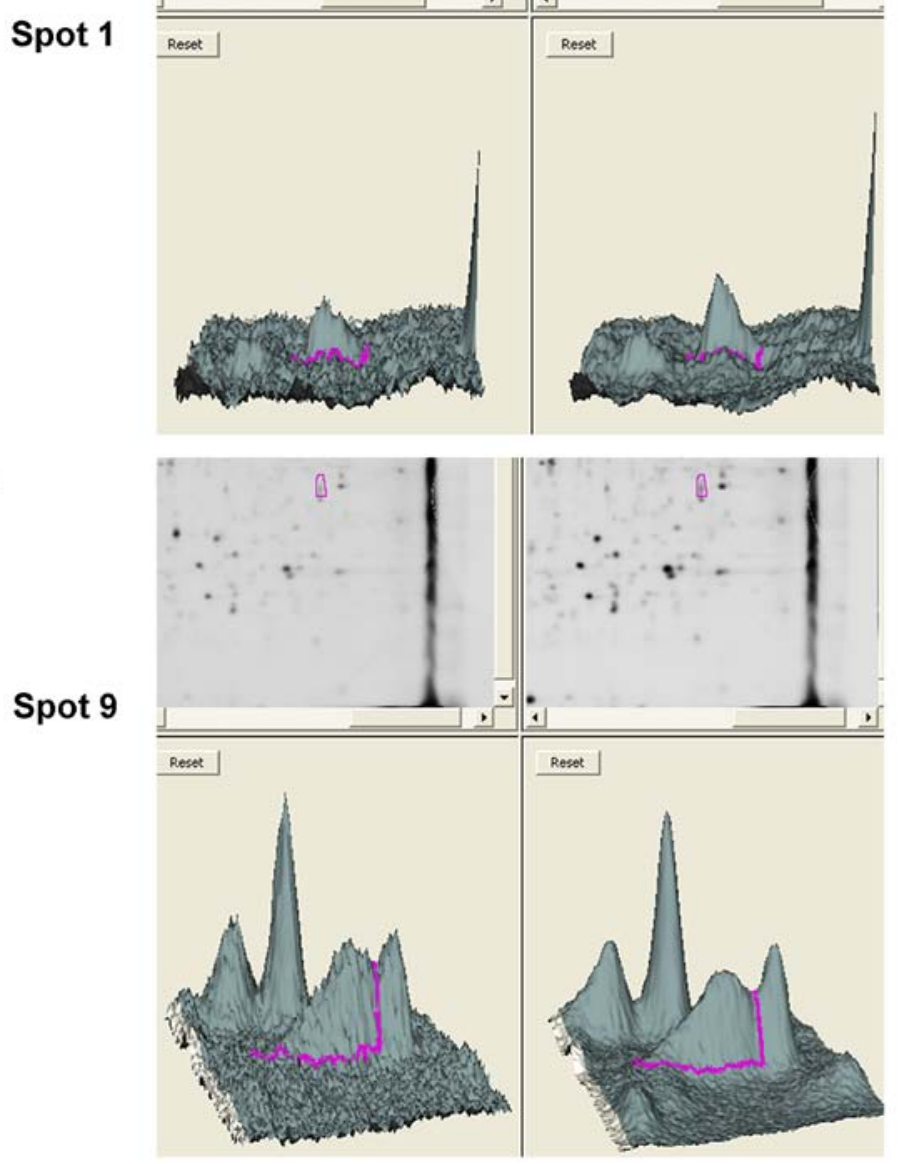

Avg ratio

Cy5

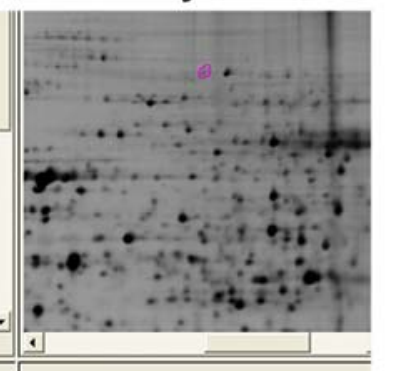

1.15

Figure 5. Examples of DeCyder software generated three-dimensional (3D) images of the species that either increased or decreased in amount in 293T cells expressing ubiquitin-specific protease 13 (USP13). Three-dimensional peaks representing spots of interest (master numbers on the left) in each panel set are circled in red. The average ratio for each spot is indicated on the right. (A) Spot 1 displayed an average 1.15-fold increase in abundance compared with the samples of USP13 (labeled with Cy5) and USP13 C345S (labeled with Cy3). (B) Spot 9 displayed an average -1.20-fold decrease in abundance compared with the samples of USP13 (labeled with Cy5) and EGFP (labeled with Cy3).

protein extracts were examined by western blot analysis to detect the expression of endogenous vinculin. The results from 3 independent experiments revealed that the overexpression of USP13, but not the enzyme-dead mutant USP13 C345S and the empty vector, led to a prominent increase in the protein levels of vinculin (Fig. 6B and C). On the contrary, the vinculin transcript levels were not significantly altered in the cells following the overexpression of USP13 (Fig. 6A). These results suggest that USP13 upregulates vinculin protein levels, but not the mRNA levels, through an enzymatically mediated mechanism.

\section{Discussion}

In the present study, the USP13 gene was identified from $293 \mathrm{~T}$ cells, and the solubility of GST-USP13 recombinant protein was found to be improved significantly at a lower temperature $\left(15^{\circ} \mathrm{C}\right)$. The USP cleavage assay using GST-Ub52 and Ub-Met- $\beta$-gal as model substrates in vitro also revealed that USP13 had no detectable DUB activity. In addition, a proteomics approach was taken by using 2D-DIGE to detect cellular proteins with significantly altered expression levels in 293T cells following the overexpression of USP13 or its C345S mutant (the catalytically inactive form) for the first time, to the best of our knowledge. Several proteins were detected and identified using LC-MS/MS. Among the identified proteins, the vinculin protein level was upregulated by USP13 through an enzymatically mediated mechanism, which was confirmed by western blot analysis and RT-qPCR.

Attempts were made to purify and improve the solubility of the fusion protein GST-USP13 for further analyses. Commonly, lowering the temperature improves the solubility of recombinant proteins (30-32) as cell processes that involve transcription, 

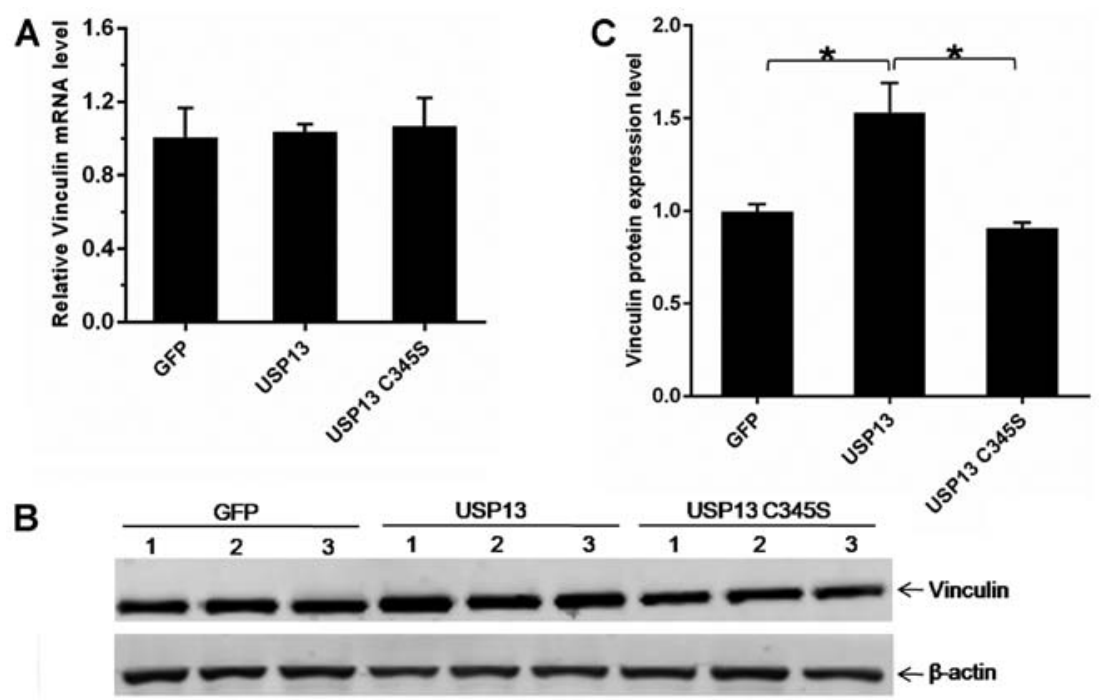

Figure 6. Ubiquitin-specific protease 13 (USP13) upregulates vinculin protein levels but not the mRNA level. (A) RT-qPCR analysis of the relative mRNA level of vinculin with the expression of USP13 and normalized to that of $\beta$-actin. Data are the means \pm standard error of the mean (SEM) from 3 independent experiments and analyzed by the Student's t-test. (B) Western blot analysis of vinculin protein levels with the expression of USP13. Three independent experiments were performed, and representative results are shown. (C) The vinculin protein levels in the panel were measured and represented in the same graph, $\beta$-actin was used for normalization. ${ }^{*} \mathrm{P}<0.05$ by one-way ANOVA. Data are the means \pm SEM of triplicates from 3 independent experiments.

translation and cell division slow down (33). In addition, due to reducing the activities of most proteases at a lower temperature, recombinant proteins GST-USP13 are protected from attack by other proteases that cause protein degradation and formation of inclusion bodies (21). As shown in Fig. 1, when the GST-USP13 fusion proteins were expressed at $15^{\circ} \mathrm{C}$, the solubility of GST-USP13 recombinant protein improved significantly.

Twotypes of model substrates, GST-Ub52 andUb-Met- $\beta$-gal, were used in this study to detect the DUB activity of USP13 for the first time (Fig. 2B and D). Neither USP13 nor C345S mutant was found to have detectable activity. GST-Ub52 and Ub-Met- $\beta$-gal, the general model substrates, are often used to detect the activity of DUBs $(7,25,27)$. The expression levels of USP13 are different for the two different types of substrates due to the different expression vectors. Thus, two different types of model substrates were used to detect the DUB activity of USP13. The present findings were in partial agreement with previous studies. Catic et al (16) discovered that USP13 reacts with ISG15VS rather than with UbVME, which provided the first biochemical evidence for the protease activity of USP13. In a recent study, USP13 was found to possess a very weak deubiquitinating activity using Ub-AMC as a substrate, but a high concentration of GST-USP13 $(500 \mathrm{nM})$ is required (14). However, the hydrolytic activities of recombinant GST-fused USP13 (100 nM) were also measured using Ub-AMC as a substrate, and no deubiquitinating activity was detected for USP13 (data not shown). The reasons for the failure may include fusion protein cleavage, low protein concentration or low protein purity.

The types and lengths of ubiquitinated proteins varied among species (34). Therefore, each DUB member may have different substrate specificity, but this specificity cannot be determined by using model substrates.

In addition to studies on the enzymatic activity of USP13, elucidating the substrate specificities of USP13 is vital to understanding the biological function of the protein. The
2D-DIGE technology is now recognized as a very powerful tool to address changes related to a variety of effects on the cellular proteome. The method allows detecting not only the changes in protein quantity but also post-translational modifications that change the charge or size of the protein (35). When coupled to LC-MS/MS, 2D-DIGE can be used to identify proteins that change in structure or concentration under specific conditions. This technology seems applicable to identifying putative new substrates for USP13, as USP13 generally upregulates substrate protein levels by stabilizing proteins via deubiquitination. In this study, 2D-DIGE and LC-MS/ MS were used to identify cellular proteins with significantly altered expression levels in 293T cell lines after the expression of USP13. Several proteins displayed altered expression levels after the overexpression of USP13 compared with that of EGFP (Fig. 3). However, when comparing the samples after the overexpression of USP13 and USP13 C345S, only vinculin exhibited increased expression levels (Fig. 4). These results suggest that the regulation of the remaining proteins whose expression level altered after the overexpression of USP13, such as THOP1, CPSF3 and Annexin by USP13 may not require USP13 deubiquitinase activity. This is simlar to the effect of USP13 on Siah2, which is not mediated by its isopeptidase activity (18). Future studies are warranted to determine the molecular mechanisms through which USP13 regulates the levels of these proteins.

Although the 2D-DIGE technology allows the detection of very small changes in spot intensity, it has some limitations. First, some overlapping of protein spots due to similarities in molecular mass and $\mathrm{p} I$ is possible. Second, the 2D-DIGE approach allows the detection of proteins that are expressed at relatively high levels. Hence, potentially important substrate proteins that are expressed at levels below the detection limit of 2D-DIGE may be missed. These limitations may explain why the present study failed to identify the known USP13 substrates MITF (21) and PTEN (23,17-19). For example, 
MITF is a member of the basic helix-loop-helix-leucine zipper transcription factor family and a key regulator of development and survival for melanocytes as well as melanomas (36). MITF is usually highly expressed in the melanoma cell lines (21).

In this study, 2D-DIGE was used to explore whether vinculin is upregulated by USP13. Vinculin is a $116-\mathrm{kDa}$ cytoskeletal protein that is involved in the linkage of integrin adhesion molecules to the actin cytoskeleton (37). In a previous study, UCH-L1, a DUB that removes $\mathrm{Ub}$ from Ub precursor proteins and hydrolyzes Ub carboxyl-terminal esters and amides (38), was found to be involved in regulating focal adhesions and adherens junctions, which is supported by co-immunoprecipitation with key components of these complexes, including FAK, paxillin, p120-catenin, $\beta$-catenin, and vinculin (39). Furthermore, vinculin is regularly co-precipitated by the wild-type and mutant UCH-L1 after mild cross-linking. However, the deubiquitylase that regulates vinculin polyubiquitylation and protein stability has not yet been reported. The present study revealed that vinculin is upregulated following the overexpression of USP13 and thereby provides a molecular clue as to whether USP13 is responsible for the deubiquitination of vinculin. Future studies are warranted to determine the ubiquitination of vinculin in 293T cell lines and whether USP13 deubiquitinates vinculin polyubiquitylation and stabilizes vinculin protein.

However, regardless of the aforementioned shortcomings, it is believed that the 2D-DIGE approach provides a useful avenue to identify new USP substrates, provided that sufficient follow-up validation studies are performed.

\section{Acknowledgements}

This study was supported by the Natural Science Foundation of Hebei Province (no. H2015206406). The authors highly appreciate Dr Man Li for contributing to mass spectrometry analysis.

\section{References}

1. Kim YK, Kim YS, Yoo KJ, Lee HJ, Lee DR, Yeo CY and Baek KH: The expression of Usp 42 during embryogenesis and spermatogenesis in mouse. Gene Expr Patterns 7: 143-148, 2007.

2. Quesada V, Díaz-Perales A, Gutiérrez-Fernández A, Garabaya C, Cal S and López-Otín C: Cloning and enzymatic analysis of 22 novel human ubiquitin-specific proteases. Biochem Biophys Res Commun 314: 54-62, 2004.

3. Baek SH, Park KC, Lee JI II, Kim KI II, Yoo YJ, Tanaka K, Baker RT and Chung CH: A novel family of ubiquitin-specific proteases in chick skeletal muscle with distinct $\mathrm{N}$ - and C-terminal extensions. Biochem J 334: 677-684, 1998.

4. D'Andrea A and Pellman D: Deubiquitinating enzymes: a new class of biological regulators. Crit Rev Biochem Mol Biol 33: 337-352, 1998.

5. Wing SS: Deubiquitinating enzymes - the importance of driving in reverse along the ubiquitin-proteasome pathway. Int J Biochem Cell Biol 35: 590-605, 2003.

6. Everett RD, Meredith M, Orr A, Cross A, Kathoria M and Parkinson J: A novel ubiquitin-specific protease is dynamically associated with the PML nuclear domain and binds to a herpesvirus regulatory protein. EMBO J 16: 1519-1530, 1997.

7. Zhang W, Tian QB, Li QK, Wang JM, Wang CN, Liu T, Liu DW and Wang MW: Lysine 92 amino acid residue of USP46, a gene associated with 'behavioral despair' in mice, influences the deubiquitinating enzyme activity. PLoS One 6: e26297, 2011.

8. Dang LC, Melandri FD and Stein RL: Kinetic and mechanistic studies on the hydrolysis of ubiquitin C-terminal 7-amido-4-methylcoumarin by deubiquitinating enzymes. Biochemistry 37 : 1868-1879, 1998.
9. Yin ST, Huang H, Zhang YH, Zhou ZR, Song AX, Hong FS and Hu HY: A fluorescence assay for elucidating the substrate specificities of deubiquitinating enzymes. Biochem Biophys Res Commun 416: 76-79, 2011.

10. Timms KM, Ansari-Lari MA, Morris W, Brown SN and Gibbs RA: The genomic organization of isopeptidase T-3 (ISOT-3), a new member of the ubiquitin specific protease family (UBP). Gene 217: 101-106, 1998.

11. Stein RL, Chen Z and Melandri F: Kinetic studies of isopeptidase T: modulation of peptidase activity by ubiquitin. Biochemistry 34: 12616-12623, 1995.

12. Lacombe T and Gabriel JM: Further characterization of the putative human isopeptidase T catalytic site. FEBS Lett 531: 469-474, 2002.

13. Reyes-Turcu FE, Horton JR, Mullally JE, Heroux A, Cheng X and Wilkinson KD: The ubiquitin binding domain ZnF UBP recognizes the $\mathrm{C}$-terminal diglycine motif of unanchored ubiquitin. Cell 124: 1197-1208, 2006.

14. Zhang YH, Zhou CJ, Zhou ZR, Song AX and Hu HY: Domain analysis reveals that a deubiquitinating enzyme USP13 performs non-activating catalysis for Lys63-linked polyubiquitin. PLoS One 6: e29362, 2011.

15. Bonnet J, Romier C, Tora L and Devys D: Zinc-finger UBPs: regulators of deubiquitylation. Trends Biochem Sci 33: 369-375, 2008

16. Catic A, Fiebiger E, Korbel GA, Blom D, Galardy PJ and Ploegh HL: Screen for ISG15-crossreactive deubiquitinases. PLoS One 2: e679, 2007.

17. Liu Y, Soetandyo N, Lee JG, Liu L, Xu Y, Clemons WM Jr and Ye Y: USP13 antagonizes gp78 to maintain functionality of a chaperone in ER-associated degradation. eLife 3: e01369, 2014.

18. Scortegagna M, Subtil T, Qi J, Kim H, Zhao W, Gu W, Kluger H and Ronai ZA: USP13 enzyme regulates Siah2 ligase stability and activity via noncatalytic ubiquitin-binding domains. J Biol Chem 286: 27333-27341, 2011.

19. Yeh HM, Yu CY, Yang HC, Ko SH, Liao CL and Lin YL: Ubiquitin-specific protease 13 regulates IFN signaling by stabilizing STAT1. J Immunol 191: 3328-3336, 2013.

20. Chen M, Gutierrez GJ and Ronai ZA: Ubiquitin-recognition protein Ufd1 couples the endoplasmic reticulum (ER) stress response to cell cycle control. Proc Natl Acad Sci USA 108: 9119-9124, 2011.

21. Zhao X, Fiske B, Kawakami A, Li J and Fisher DE: Regulation of MITF stability by the USP13 deubiquitinase. Nat Commun 2: 414, 2011.

22. Liu J, Xia H, Kim M, Xu L, Li Y, Zhang L, Cai Y, Norberg HV, Zhang T, Furuya T, et al: Beclin1 controls the levels of p53 by regulating the deubiquitination activity of USP10 and USP13. Cell 147: 223-234, 2011.

23. Zhang J, Zhang P, Wei Y, Piao HL, Wang W, Maddika S, Wang M, Chen D, Sun Y, Hung MC, et al: Deubiquitylation and stabilization of PTEN by USP13. Nat Cell Biol 15: 1486-1494, 2013.

24. Geng J, Huang X, Li Y, Xu X, Li S, Jiang D, Liang J, Jiang D, Wang C and Dai H: Down-regulation of USP13 mediates phenotype transformation of fibroblasts in idiopathic pulmonary fibrosis. Respir Res 16: 124, 2015.

25. Liu YL, Zheng J, Tang LJ, Han W, Wang JM, Liu DW and Tian QB: The deubiquitinating enzyme activity of USP22 is necessary for regulating HeLa cell growth. Gene 572: 49-56, 2015.

26. Tian QB, Okano A, Nakayama K, Miyazawa S, Endo S and Suzuki T: A novel ubiquitin-specific protease, synUSP, is localized at the post-synaptic density and post-synaptic lipid raft. J Neurochem 87: 665-675, 2003.

27. Tang LJ, Li Y, Liu YL, Wang JM, Liu DW and Tian QB: USP12 regulates cell cycle progression by involving c-Myc, cyclin D2 and BMI-1. Gene 578: 92-99, 2016.

28. Bradford MM: A rapid and sensitive method for the quantitation of microgram quantities of protein utilizing the principle of protein-dye binding. Anal Biochem 72: 248-254, 1976.

29. Tang W: Quantitative analysis of plasma membrane proteome using two-dimensional difference gel electrophoresis. Methods Mol Biol 876: 67-82, 2012.

30. Shirano Y and Shibata D: Low temperature cultivation of Escherichia coli carrying a rice lipoxygenase L-2 cDNA produces a soluble and active enzyme at a high level. FEBS Lett 271: 128-130, 1990.

31. Kataeva I, Chang J, Xu H, Luan CH, Zhou J, Uversky VN, Lin D, Horanyi P, Liu ZJ, Ljungdahl LG, et al: Improving solubility of Shewanella oneidensis MR-1 and Clostridium thermocellum JW-20 proteins expressed into Esherichia coli. J Proteome Res 4: 1942-1951, 2005. 
32. Volontè F, Marinelli F, Gastaldo L, Sacchi S, Pilone MS, Pollegioni L and Molla G: Optimization of glutaryl-7-aminocephalosporanic acid acylase expression in E. coli. Protein Expr Purif 61: 131-137, 2008

33. Chou CP: Engineering cell physiology to enhance recombinant protein production in Escherichia coli. Appl Microbiol Biotechnol 76: 521-532, 2007.

34. Larsen CN, Krantz BA and Wilkinson KD: Substrate specificity of deubiquitinating enzymes: ubiquitin C-terminal hydrolases. Biochemistry 37: 3358-3368, 1998.

35. Kolkman A, Dirksen EH, Slijper M and Heck AJ: Double standards in quantitative proteomics: direct comparative assessment of difference in gel electrophoresis and metabolic stable isotope labeling. Mol Cell Proteomics 4: 255-266, 2005.

36. Levy C, Khaled M and Fisher DE: MITF: master regulator of melanocyte development and melanoma oncogene. Trends Mol Med 12: 406-414, 2006.
37. Ziegler WH, Liddington RC and Critchley DR: The structure and regulation of vinculin. Trends Cell Biol 16: 453-460, 2006. 38. Das C, Hoang QQ, Kreinbring CA, Luchansky SJ, Meray RK, Ray SS, Lansbury PT, Ringe D and Petsko GA: Structural basis for conformational plasticity of the Parkinson's disease-associated ubiquitin hydrolase UCH-L1. Proc Natl Acad Sci USA 103: 4675-4680, 2006.

39. Frisan T, Coppotelli G, Dryselius R and Masucci MG: Ubiquitin C-terminal hydrolase-L1 interacts with adhesion complexes and promotes cell migration, survival, and anchorage independent growth. FASEB J 26: 5060-5070, 2012. 